\title{
Comparative study of strong anion exchange hypercrosslinked poly(HEMA-co-EGDMA-co-VBC) and strong anion exchange poly(Styrene-co-EGDMA-co- VBC): Synthesis and characterization
}

\author{
N. Abdullah, ${ }^{1,2 *}$ M. Z. Yahya ${ }^{1}$, R. Mahmod ${ }^{1}$.
}

${ }^{1}$ Faculty of Chemical Engineering \& Natural Resources, Universiti Malaysia Pahang. ${ }^{2}$ Rare Earth Research Centre (RERC), Universiti Malaysia Pahang, 26300 Gambang, Pahang, Malaysia. Article Info: Submitted on March 20, 2017, Accepted on June 20, 2017.

\begin{abstract}
Strong anion exchange hypercrosslinked of poly(HEMA-co-EGDMA-co-VBC) (PHEV) and strong anion exchange hypercrosslinked of poly(styrene-co-EGDMA-co-VBC) (PSEV) were prepared via amination reaction with dimethylbutyl amine (DMBA) as the amination agent in dried toluene. The resins were characterized by SEM, FTIR, BET and elemental analysis. SEM results showed that there was no significant change of the particles shape after amination reaction for both strong anion exchange hypercrosslinked PHEV and strong anion exchange hypercrosslinked PSEV. Specific surface area (SSA) of strong anion exchange hypercrosslinked PHEV increase from $1035 \mathrm{~m}^{2} / \mathrm{g}$ to $1090 \mathrm{~m}^{2} / \mathrm{g}$ when amination reaction take place. However, SSA of strong anion exchange hypercrosslinked PHEV decreased from $124 \mathrm{~m}^{2} / \mathrm{g}$ to $27 \mathrm{~m}^{2} / \mathrm{g}$. From FTIR spectra, significant drop occurred at $1265 \mathrm{~cm}^{-1}$ band after amination reaction of strong anion exchange hypercrosslinked PSEV but SSA of strong anion exchange hypercrosslinkedPHEV showed slightly significant drop. Elemental analysis showed that nitrogen content of strong anion exchange hypercrosslinked PHEV significantly increase from $0.10 \%$ to $1.46 \%$ resulted to high IEC value. Meanwhile, nitrogen content in strong anion exchange hypercrosslinked PSEV only showed small increment in nitrogen content from $0.36 \%$ to $0.63 \%$ led to low IEC value.
\end{abstract}

Keywords: Hypercrosslinked polymer; Amination reaction; Strong anion exchange resin.

\section{Introduction}

Since years ago, considerable interest has been developed in the synthesis of ion-exchange resin having selective properties and containing selective functional groups.The main application for strong anion resin is in separation process. Quaternary ammonium is ammonium cation with four alkyl group or aryl group attached to the nitrogen atom and it carries positive charge that suitable for many applications and being known as strong anion resin. Many studies showed that positive charges in resin have potential to be used in many applications. For example, in sulfur removal, ${ }^{1}$ phosphorus removal in wastewater, ${ }^{2}$ separation of actinides elements in sediments and biological materials, ${ }^{3}$ and even be used to decrease the acid value of crude low-calorie cocoa butter to an acceptable level. ${ }^{4}$ Most of the strong anion resins used in the studies is commercially available.

Generally, conventional copolymers sorbent available commercially such as D202 anion exchange resin and Amberlite IRA 420 are normally based on styrenedivinylbenzene (DVB) copolymer which these resins show hydrophobic characteristic. This hydrophobicity is more efficient in the retention of non-polar compounds.
Meanwhile, hydrophilic polymers contain polar functional groups and it is more useful for retention of polar compounds. ${ }^{5,6}$ Development of resin containing hydrophilic characteristic have been done since a few year ago and have received great attention from many researchers. $^{7,8}$

Commercially ion exchange resin available also can be either gel-type or macroporous structure and usually have hydrophobic characteristics. Drawback of such ion-exchange resin is that it has limitedhydrophilic character which is in separation application, such resin useful for retention non-polar compound only and have low specific surface area (SSA). Gel-type resin have higher (SSA) (>1000 m²/g)[1] $]^{9,10}$ and macroporous structure have lower than $600 \mathrm{~m}^{2} / \mathrm{g}$. ${ }^{11}$

Hypercrosslinked polymers have a great attention due to they have a few striking features such as it is display high specific surface area, very low density, chemical and mechanical stability. Thus, they were classified as a new generation of porous polymer and replace the conventional porous polymer since discovery by Davankov late 1960s. ${ }^{12}$ Because of the unusual features of hypercrosslinked polymer, they have a vast opportunity in terms of applications for example in chromatography columns, separation and also used as 


\section{Chemical Engineering Research Bulletin 19(2017) 96-101}

starting material/precursor to synthesis ion exchanger resin. $^{11}$

Starting material upon hypercrosslinking reaction or functionalization reaction can be synthesized in many ways. The polymerization techniques included dispersion polymerization and suspension polymerizationcan be carried out as the preparation of precursor resulting in different size and morphology.In this comparative study, we describe how the precursors derived from two different polymerization techniques effects on stronganion exchange hypercrosslinked resin.

\section{Materials and Methods}

\section{Materials}

The reagent used for non-aqueous dispersion (NAD) polymerization and suspension polymerization (SP)were styrene (99\% grade) supplied by Aldrich (Steinheim, Germany), 4-vinylbenzyl chloride (VBC) ( $95 \%$ grade) supplied by Fluka (Steinheim, Germany), methanol ( $99.7 \%$ grade), ethanol (95\% grade), poly(Nvinylpyrrolidone) 55 (PVP) (MW 55,000), Triton X305 and 2,2'-azobisisobutyronitrile (AIBN) (97\% grade)was supplied by BDH (Poole, UK) and purified by recrystallization from methanol, 2hydroethylmethacrylate (HEMA) (97\% grade) contains $200 \mathrm{ppm}$ monomethyl ether hydroquinone and benzoyl peroxide (BPO) $(75 \%$ grade)purified by recrystallization from chloroform were obtained from Sigma Aldrich, Toluene (99.5\% grade) was obtained from HmBG Chemical and distilled water. Inhypercrosslinking reactions, the reagents used were anhydrous 1,2-dichloroethane (DCE) (99\% grade), and Ferum (III) chloride, $\left(\mathrm{FeCl}_{3}\right) \quad(98 \%$ grade), were supplied by Sigma-Aldrich.Dried toluene (99.8\% grade), dimethylbutylamine (DMBA) (99.8\% grade), and methanol (99.8\% grade), from Sigma-Aldrich, sodium hydrogen carbonate ( $99.8 \%$ grade) supplied by VWR and distilled water were used in amination reaction.All of them were used as received.

\section{Resin preparation}

In precursor polymerization, the spherical particles used as swellable precursors in the production of strong anion exchange resin, were synthesized using non-aqueous dispersion polymerisation (NAD) and suspension polymerisation (SP). ${ }^{9,13}$ In NAD polymerizations, all of the styrene, stabilizer, costabilizer, initiator, and half of the VBC and ethanol were added into a $500 \mathrm{~mL}$ five-necked, roundbottomed flask fitted with an overhead stirrer, condenser and nitrogen inlet. The flask was then placed into an oil bath set at $70{ }^{\circ} \mathrm{C}$, and stirred. EGDMA and the second portion of VBC were dissolved in the remaining half of ethanol at $70{ }^{\circ} \mathrm{C}$ under nitrogen inlet. One hour after polymerization had begun, EGDMA and VBC were added into the reaction vessel and reaction was carried out until 24 hours. The polymer particles that formed were washed twice with ethanol and twice with methanol by centrifugation. The particles were filtered using vacuum filtration on a $0.22 \mu \mathrm{m}$ nylon membrane filter and dried overnight in oven at $40{ }^{\circ} \mathrm{C}$.

In SP, dispersion medium was prepared by dissolving PVP in distilled water. Then toluene was mixed with HEMA, BPO, EDGMA, and VBC. The mixture then being transferred into the dispersion medium placed in $250 \mathrm{~mL}$ five-necked, round-bottomed flask fitted with a condenser and nitrogen inlet and magnetic stirrer. The reactor was flushed by nitrogen for 30 minutes before the flask was put into the oil bath. Polymerization process was conducted at $75{ }^{\circ} \mathrm{C}$ for 6 hours with constant stirring speed at $300 \mathrm{rpm}$.After polymerization, the microsphere then washed by distilled water and methanol before dry in oven at 40 ${ }^{\circ} \mathrm{Cfor} 24$ hours.

The hypercrosslinking reactions the precursor particles $(\sim 1.2 \mathrm{~g})$ were added into a round- bottomed flask containing DCE $(40 \mathrm{~mL})$ and left to swell fully under nitrogen for 1 hour. Then, $\mathrm{FeCl}_{3}$ (in a 5\% molar excess relative to chloromethyl groups) suspended in DCE $(40 \mathrm{~mL})$ was added. The mixture was then heated rapidly to $80{ }^{\circ} \mathrm{C}$ and reaction was carried out for 18 hours. After cooling, the hypercrosslinked particles were filtered and washed with $\mathrm{MeOH}$, and then washed several times with aqueous $\mathrm{HNO}_{3}(\mathrm{pH} 2)$. Then, the hypercrosslinked polymer were extracted overnight with acetone in a Soxhlet extractor and dried overnight in vacuo at $40{ }^{\circ} \mathrm{C} .{ }^{9}$

In typical amination reaction, hypercrosslinked polymer $(\sim 0.5 \mathrm{~g})$ and dried toluene $(80 \mathrm{~mL})$ were placed in a round bottomed- flask $(100 \mathrm{~mL})$ and the mixture left under $\mathrm{N}_{2}$ for 1 hour to wet the beads. Then, dimethylbutyl amine (DMBA) (in 5 molar excess relative to $-\mathrm{CH}_{2} \mathrm{Cl}$ ) was added into the solution. The mixture then was heated rapidly to $85^{\circ} \mathrm{C}$ and kept this temperature for $18 \mathrm{~h}$. The aminated beads were filtered and washed with toluene, $\mathrm{MeOH}, \mathrm{MeOH}: \mathrm{H}_{2} \mathrm{O}$ and then washed several times with aqueous $5 \%(\mathrm{w} / \mathrm{v})$ $\mathrm{NaHCO}_{3}$ and water. Then the aminated beads were then extracted overnight with acetone in a soxhlet extractor before drying overnight in oven at $40{ }^{\circ} \mathrm{C}$.

\section{Characterization}

The morphology of the polymer particle was captured by using CARL ZEISS Scanning Electron Microscopy (SEM). The SEM images of poly(styrene-co-EGDMA$c o$-VBC) particles were captured using SEM at 3.95X, $2.48 \mathrm{kX}$, and $1.64 \mathrm{kX}$ magnification. The SEM images of poly(HEMA-co-EGDMA-co-VBC) particles were captured using SEM at60X and 50X magnification. The functional group presence in the particles was identified using Nicolet iS50 (Thermo Scientific) Fourier transforms infrared spectroscopy analysis 


\section{Chemical Engineering Research Bulletin 19(2017) 96-101}

(FTIR). Specific surface area of the hypercrosslinked polymer was characterized by using a BrunauerEmmett-Teller (BET) (Micromeritics ASAP 2010 BET ANALYZER).The amounts of nitrogen presence in the particles were determined using elemental analysis equipment from Elementar Germany.

\section{Results and Discussion}

\section{Morphology and specific surface area (SSA) of particles}

Figure 1 and Figure 2 shows morphology of strong anion exchange resin. As can be seen in Figure 1a and $1 \mathrm{~b}$, hypercrosslinked poly(styrene-co-EGDMA-coVBC) (PSEV) particles cannot retain the original shape upon hypercrosslinking reaction. This occurred due to amount of crosslinker that presence in the backbone of polymer very small resulting to the particles aggregated. However, there were no significant changes observed on hypercrosslinked particles before and after amination reaction take place (Figure $1 \mathrm{~b}$ and 1c). The presence of high degree of crosslinking in the backbone of polymer formed during hypercrosslinking reaction provide extra rigidity to the particles. Therefore, when the solvent is removed from the system, the collapse of the hypercrosslinked PSEV particles been avoided. ${ }^{14}$

Figure 2 shows images of strong anion exchange hypercrosslinked PHEV. In all reaction stages (SP to amination reaction), the particles managed to retain their shape. The hypercrosslinked polymer can retain the original shape because it contributed by the presence of high amount of crosslinker during precursor synthesis. Similar results have been obtained by other researchers. ${ }^{11,13}$ Meanwhile, structure of strong anion exchange hypercrosslinked PHEV further strengthen by the presence of extra crosslinked chain formed during hypercrosslinking reaction. After amination process, the surface of the particles become smoother and the pore became less prominent at the surface (Figure 2c).

Table 1 depicted the specific surface area (SSA) before and after amination for both strong anion exchange hypercrosslinked PSEV and PHEV. SSA for precursor in both cases around $\sim 5 \mathrm{~m}^{2} / \mathrm{g}$ which prove that the precursors are non-porous in dry state. For PSEV, the precursors were easier to swell in solvent during hypercrosslinking reaction compared to macroporous PHEV due to small amount of crosslinker presence in precursor.This causing more chloromethyl group being transformed to methylenebridge and subsequently formed pores. ${ }^{15}$ However, for PHEV, the precursor containing high amount of crosslinker resulting the particles more rigid and less swell in solvent. This contributes to less conversion of chloromethyl group to methylene bridges. Therefore, hypercrosslinked PSEV have more SSA compared to hypercrosslinked PHEV.

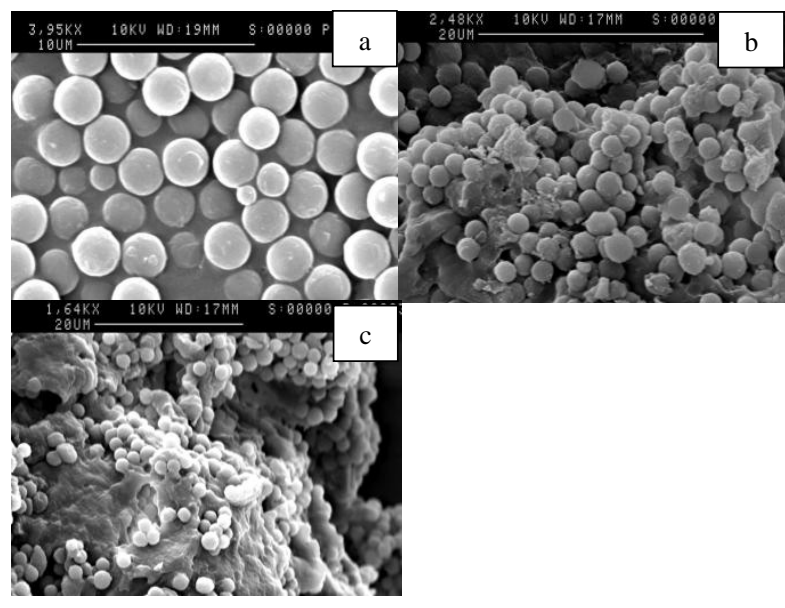

Figure 1: SEM imagesof PSEV: a) PSEV precursor at $3.95 \mathrm{kX}$ magnification, b) hyper cross linked particles at $2.48 \mathrm{kX}$ magnification, and c) hyper cross linked aminated particles at $1.64 \mathrm{kX}$ magnification.

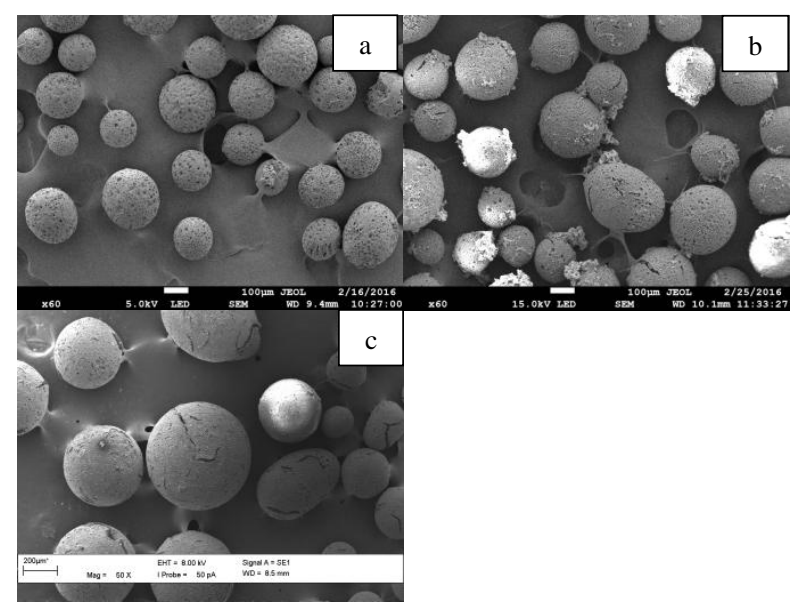

Figure 2: SEM images of PHEV: a) PHEV precursor at $60 \mathrm{X}$ magnification, b) hyper cross linked particles at $60 \mathrm{X}$ magnification, and c) hyper cross linked aminated particles at 50X magnification.

The SSA before amination strong anion exchanges hypercrosslinked PSEV exceeding $1000 \mathrm{~m}^{2} / \mathrm{g}$. After amination, SSA slightly increasing probably it arises from additional crosslinking which contribute to increasing in SSA instead of the porous structure of strong anion exchange hypercrosslinked polymer itself. Similar result was also reported in literature. ${ }^{16-18}$ However, for strong anion exchange hypercrosslinked PHEV, the SSA decreased significantlyfrom $124 \mathrm{~m}^{2} / \mathrm{g}$ to $27 \mathrm{~m}^{2} / \mathrm{g}$. This might be caused by the bulky functional group arise from DMBA block the pore of the particles. Despite of decreasing in SSA, smoother surface of the resin also can be seen in Figure 2c which it most probably due to the particle poreshave been covered by DMBA. 


\section{Chemical Engineering Research Bulletin 19(2017) 96-101}

Table 1: BET analysis of hypercrosslinked PSEV a hypercrosslinked PHEV resin before and after amination.

\begin{tabular}{|c|c|c|c|c|}
\hline Sample & $\begin{array}{c}\mathrm{SSA}^{\mathrm{a}} \\
\left(\mathrm{m}^{2} / \mathrm{g}\right)\end{array}$ & $\begin{array}{c}\mathrm{SSA}^{\mathrm{b}} \\
\left(\mathrm{m}^{2} / \mathrm{g}\right)\end{array}$ & $\mathrm{SSA}^{\mathrm{c}}\left(\mathrm{m}^{2} / \mathrm{g}\right)$ & $\begin{array}{c}\text { Yield } \\
(\%)\end{array}$ \\
\hline PSEV & $\sim 5$ & 1035 & 1090 & 65.00 \\
\hline PHEV & $\sim 5$ & 124 & 27 & 78.19 \\
\hline
\end{tabular}

${ }^{\text {a }} \mathrm{SSA}$ of precursor

${ }^{\mathrm{b}} \mathrm{SSA}$ of hypercrosslinked PSEV and PHEV

${ }^{\mathrm{c}} \mathrm{SSA}$ of strong anion exchange hypercrosslinked PSEV and PHEV

\section{FTIR Analysis}

The spectra of strong anion exchange hypercrosslinked PSEV and PHEV before and after amination reaction are shown in Figure 3. Both polymers have characteristic absorption bands at $1265 \mathrm{~cm}^{-1}$ and 733 $\mathrm{cm}^{-1}$ arise from stretching vibrations of $\mathrm{C}-\mathrm{Cl}$ bonds and $975 \mathrm{~cm}^{-1}$ is ascribed to $\mathrm{CH}_{2}$ vibration that present in hypercrosslinked polymer before amination. ${ }^{17}$ For strong anion exchange hypercrosslinked PSEV (Figure 3a),slightly reduction at band $1265 \mathrm{~cm}^{-1}$ can be observed. This probably because of chlorine residues in hypercrosslinked polymer decreased (high SSA, Table 1) resulting low conversion of chlorine groups to amine groups. However, for strong anion exchange hypercrosslinked PHEV (Figure $3 b$ ) the reduction in intensity at band $1265 \mathrm{~cm}^{-1}$ clearly can be observed due to the high residue chlorine inside hypercrosslinked polymer. General speaking, the reduction of intensity at band $1265 \mathrm{~cm}^{-1}$ was due to the reaction between $\mathrm{Cl}$ groups and their adjacent $\mathrm{H}$ cause additional $\mathrm{Cl}$ loss. ${ }^{11}$ The loss of $\mathrm{Cl}$ was replaced by DMBA. In the both cases, DMBA was successfully attach at the backbone of polymers.

\section{Ion Exchange Capacity (IEC) and nitrogen content}

An increasing of nitrogen percentage was observed from hypercrosslinked polymer to strong anion exchange hypercrosslinked for PSEV and PHEV (Table 2). This was as expected which $\mathrm{Cl}$ was replaced by DMBA thus provide increasing in nitrogen content. Nitrogen content slightly increased from $0.36 \%$ to $0.63 \%$ after amination reaction for strong anion exchange hypercrosslinked PSEV. Meanwhile, for strong anion exchange hypercrosslinked PHEV, the nitrogen content significant increase from $0.10 \%$ to $1.46 \%$ (Table 2).

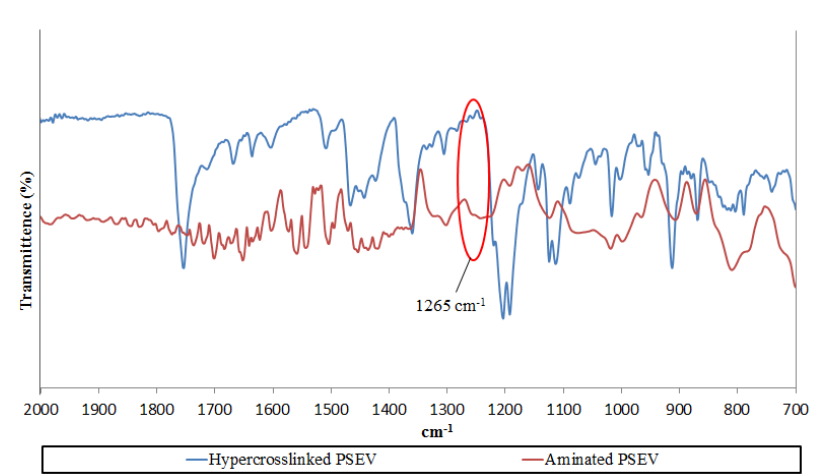

(a)

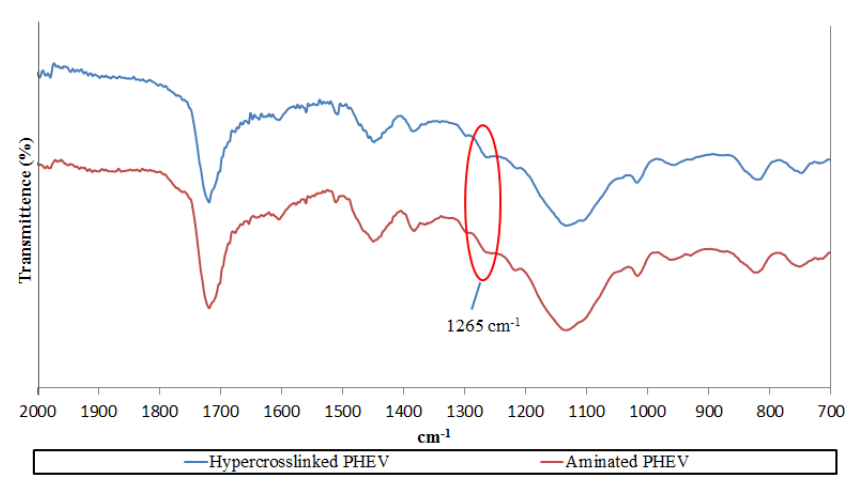

(b)

Figure 3: FTIR analysis for hypercrosslinked and aminated resin: a) PSEV; b) PHEV.

The significant increase in nitrogen content observed at strong anion exchange hypercrosslinkedPHEV probably due to high chlorine residue inside the hypercrosslinked polymer prior amination reaction. Therefore, more amine groups can take place during amination reactions. This result also in a good agreement with FTIR and SSA results of such polymer (Table 1 and Figure 3b).

The elemental results also in good agreement with the ion exchange capacity (IEC) (Table 2 ) for both strong anion exchange hypercrosslinked PHEV and PSEV. High amount of nitrogen indicates that more ion exchange site available in the particles. Ion exchange site available for strong anion exchange hypercrosslinked PSEV was determined to be around $0.193 \mathrm{mmol} / \mathrm{g}$ while ion exchange site available for strong anion exchange hypercrosslinked PHEV was determined to be around $9.97 \mathrm{mmol} / \mathrm{g}$. The difference in IEC values was due to high amount of chloromethyl group available at hypercrosslinked PHEV to allow amine groups take place chlorine groups site during amination reaction. Therefore, more DMBA were attached to the polymeric backbone compared to strong anion exchange hypercrosslinked PSEV. Attachment of DMBA into the polymeric backbone formed positively charge nitrogen group (quarternary ammonium group) which provide ion exchange site. 


\section{Chemical Engineering Research Bulletin 19(2017) 96-101}

Table 2: Nitrogen content for both PSEV and PHEV resin (before and after amination) and their ion exchange capacity (IEC).

\begin{tabular}{cccc}
\hline Sample & \multicolumn{2}{c}{ Nitrogen content (\%) } & IEC $^{\mathrm{a}}$ \\
& Hypercrosslinked & Aminated & $(\mathrm{mmol} / \mathrm{g})$ \\
\hline PSEV & 0.36 & 0.63 & 0.193 \\
PHEV & 0.10 & 1.46 & 9.970 \\
\hline
\end{tabular}

\section{Conclusion}

From SEM, both strong anion exchange hypercrosslinked PSEV and PHEV manage to retain their shape after amination reaction. This contributed by the presence of high degree crosslinking after hypercrosslinking reaction. Meanwhile, SSA for hypercrosslinked PSEV higher than hypercrosslinked PHEV but after amination reaction SSA lower than strong anion hypercrosslinked PHEV. From FTIR spectra, quarternary ammonium groups were successful attached at the polymer backbonein both cases.Strong anion hypercrosslinked PHEV also containing higher nitrogen content and IEC compared to strong anion hypercrosslinked PSEV.This prove for existence of more $\mathrm{N}^{+}$functional group at the backbone and more ion exchange site available in strong anion hypercrosslinked PHEV polymer.

\section{Acknowledgement}

The authors would like to be obliged to Universiti Malaysia Pahang for providing laboratory facilities and Ministry of Malaysia of Higher Education (MOHE) for financial assistance under Fundamental Research Grant Scheme project no. RDU140133.

\section{References}

1. J. F. Paulino and J. C. Afonso, "New strategies for treatment and reuse of spent sulfidic caustic stream from petroleum industry,"Química Nova, vol. 35, no. 9, pp. 1447-1452, 2012.

2. T. Nur, M. A. H. Johir, P. Loganathan, T. Nguyen, S. Vigneswaran and J. Kandasamy, "Phosphate removal from water using an iron oxide impregnated strong base anion exchange resin," Journal of Industrial and Engineering Chemistry, vol. 20, no. 4, pp.1301-1307, 2014.

3. S. H. Lee, J. La Rosa, J. Gastaud and P. P. Povinec, "The development of sequential separation methods for the analysis of actinides in sediments and biological materials using anionexchange resins and extraction chromatography,"Journal of radioanalytical and nuclear chemistry, vol. 263, no. 2, pp.419-425, 2005.
4. W. L. Wu, Z. Q. Tan, G. J. Wu, L. Yuan, W. L. Zhu, Y. L. Bao, L. Y. Pan, Y. J. Yang and J. X. Zheng, "Deacidification of crude low-calorie cocoa butter with liquid-liquid extraction and strong-base anion exchange resin,"Separation and Purification Technology, vol. 102, pp.163-172, 2013.

5. J. Patsias and E. Papadopoulou-Mourkidou, "Development of an automated on-line solid-phase extraction-high-performance liquid chromatographic method for the analysis of aniline, phenol, caffeine and various selected substituted aniline and phenol compounds in aqueous matrices," Journal of chromatography A, vol. 904, no. 2, pp.171-188, 2000.

6. R. Wissiack, E. Rosenberg and M. Grassbauer, "Comparison of different sorbent materials for online solid-phase extraction with liquid chromatography-atmospheric pressure chemical ionization mass spectrometry of phenols," Journal of Chromatography A, vol. 896, no. 1, pp.159-170, 2000.

7. H. Bagheri, A. Mohammadi and A. Salemi, "Online trace enrichment of phenolic compounds from water using a pyrrole-based polymer as the solidphase extraction sorbent coupled with highperformance liquid chromatography,"AnalyticaChimicaActa, vol. 513, no. 2, pp. 445-449, 2004.

8. 8.H. Bagheri and A. Mohammadi, "Pyrrole-based conductive polymer as the solid-phase extraction medium for the preconcentration of environmental pollutants in water samples followed by gas chromatography with flame ionization and mass spectrometry detection," Journal of Chromatography A, vol. 1015, no. 1, pp.23-30, 2003.

9. N. Abdullah and P. A. G. Cormack, "NonAqueous Dispersion (NAD) Polymerisation-Based Synthetic Route to Hypercrosslinked Polymer: Effect of Reaction Temperature and Solvent System on Specific Surface Area," In Advanced Materials Research, Trans Tech Publications, vol. 1134, pp. 198-202, 2016.

10. N. Fontanals, N. Miralles, N. Abdullah, A. Davies, N. Gilart and P. A. G. Cormack, "Evaluation of strong cation-exchange polymers for the determination of drugs by solid-phase extractionliquid chromatography-tandem mass spectrometry," Journal of Chromatography A, vol. 1343, pp. 55-62, 2014.

11. D. Bratkowska, N. Fontanals, F. Borrull, P. A. Cormack, D. C. Sherrington and R. M. Marcé,"Hydrophilic hypercrosslinked polymeric sorbents for the solid-phase extraction of polar contaminants from water," Journal of 


\section{Chemical Engineering Research Bulletin 19(2017) 96-101}

Chromatography A, vol. 1217, no. 19, pp.32383243, 2010.

12. V. A. Davankov, M. M. Ilyin, M. P. Tsyurupa, G. I. Timofeeva and L. V. Dubrovina, "From a Dissolved Polystyrene Coil to an IntramolecularlyHyper-Cross-Linked "Nanosponge," Macromolecules, vol. 26, no. 29, pp. 8398-8403, 1996.

13. K. Kesenci, A. Tuncel and E. Pişkin,"Swellable ethylene glycol dimethacrylatehydroxyethylmethacrylate copolymer beads," Reactive and Functional Polymers, vol. 31, no. 2, pp.137-147, 1996.

14. J. Germain, J. M. Fréchet and F. Svec, "Nanoporous polymers for hydrogen storage," Small, vol. 5, no. 10, pp.1098-1111, 2009.

15. N. Fontanals, R. M. Marcé, F. Borrull and P. A. G. Cormack, "Hypercrosslinked materials: preparation, characterisation and applications,"
Polymer Chemistry, vol. 6, no. 41, pp.7231-7244, 2015.

16. J. H. Hong, M. C. Park, S. K. Hong and B. S. Kim, "Preparation of an anion-exchange membrane by the amination of chlorinated polypropylene and polyethyleneimine at a low temperature and its ion-exchange property," Journal of Applied Polymer Science, vol. 112, no. 2, pp.830-835, 2009.

17. J. Liu, J. Yao, H. Wang and K. Y. Chan, "Fabrication of porous polymer particles with high anion exchange capacity by amination reaction in aqueous medium," Green Chemistry, vol. 8, no.4, pp.386-389, 2006.

18. $\mathrm{H} . \mathrm{Xu}$ and $\mathrm{X}$. Hu, "Preparation of anion exchangers by reductive amination of acetylated crosslinked polystyrene," Reactive and Functional Polymers, vol. 42, no. 3, pp.235-242, 1999.

\section{Available online at http://www.banglajol.info/index.php/CERB}

